\title{
Temperature characteristics of the nonlinear magnetoelectric effect
}

\author{
Burdin Dmitry, ${ }^{*}$, Ekonomov Nikolay, Chashin Dmitry and Fetisov Yury \\ Moscow Technological University (MIREA), 119454, Vernadskogo prosp., 78, Moscow, Russian Federation
}

\begin{abstract}
The temperature characteristics of the nonresonant nonlinear magnetoelectric effect in the twolayer LGT-Metglas structure at a large excitation field amplitude are studied. It is shown that the harmonics of the signal have a different course when the temperature is varied in the range from $200 \mathrm{~K}$ to $400 \mathrm{~K}$, which is explained by a change in the shape of the magnetization curve. Amplitude of alternating magnetic field affects the form of each ME voltage harmonic.
\end{abstract}

\section{Introduction}

Magnetoelectric (ME) effect in composite structures opens the prospect of creating a whole series of new devices of solid state electronics [1-2]. For the application of the composite ME structures it is important to investigate their temperature properties. Temperature characteristics of the linear ME effect in structures containg layers of PZT and amorphous alloy Metglas has been studied [3-6]. It was shown that temperature strongly affect the ME coefficient, which was explained by the concurrent variations with temperature of piezoelectric modulus of the PE layer, piezomagnetic modulus of the FM layer and mechanical coupling. Ye et al [3] also experimentally established relation between specimen size and ME signal temperature dependence.

Last few years a great interest is caused by the nonlinear ME effect in composite structures. Theory of nonlinear ME effect was developed [7, 8], and new devices based on it were suggested. In particular, new high sensitive ac and dc magnetic field sensors were described $[9,10]$. In this connection, it is interesting to investigate the temperature characteristics of the nonlinear ME effect, which has so far not been practically studied.

Due to the nonlinear field dependence of the ferromagnet magnetostriction (Fig.1), when the ME structure is excited by an alternating magnetic field with frequency $f$, various voltage harmonics with frequencies $f, 2 f, 3 f$, etc. are generated. The magnitude of the harmonics depends on the amplitude of the alternating magnetic field $h$ and the constant magnetic field $H$.

The aim of this work is to investigate the temperature dependences of the ME voltage harmonics generated by the piezoelectric-ferromagnetic (PE-FM) composite structure for different values of the amplitude $h$. For producing magnetostrictive layer an amorphous ferromagnetic alloys Metglas was chosen due to it's low saturation field and high magnetostriction. For piezoelectric layer most suitable material is lanthanum gallium tantalate due to its high temperature stability and rather high $d / \varepsilon$ ratio [11].

\section{Experiment}

\subsection{Samples and measurements}

A two-layer structure with in-plane dimensions of 20 $\mathrm{mm} \times 5 \mathrm{~mm}$ containing a $0.5 \mathrm{~mm}$ thick layer of monocrystalline lanthanum gallium tantalate (LGT) and a $20 \mu \mathrm{m}$ thick layer of amorphous magnetostrictive alloy Metglas 2605S3A with composition FeBSiC was used in measurements. The surfaces of the LGT layer have been metalized and the layers were connected by a lowtemperature soldering.

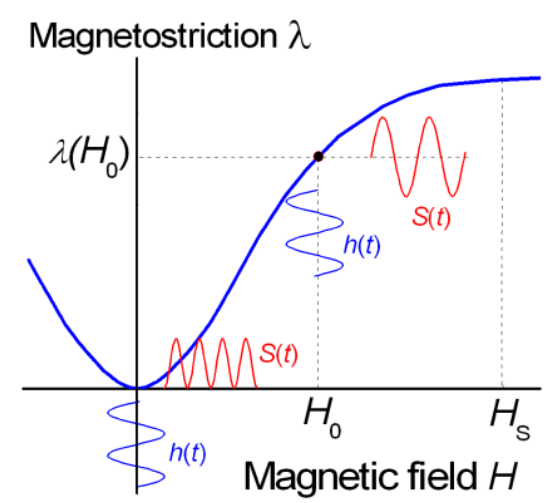

Fig. 1. Typical dependence of the FM layer magnetostriction $\lambda$ on magnetic field $H$.

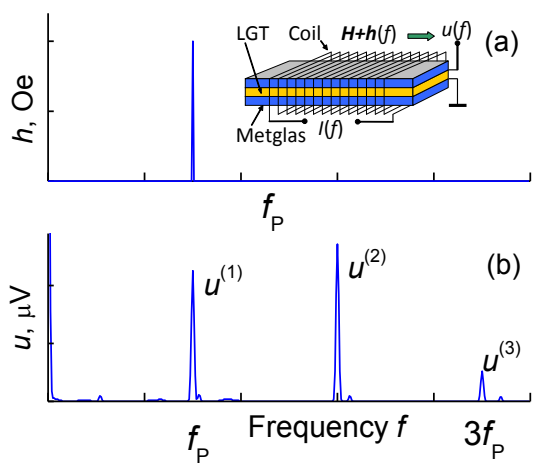

Fig. 2. Frequency spectra: (a) for pumping magnetic field and (b) for ME voltage generated by the LGT-Metglas structure.

\footnotetext{
*D.A. Burdin: phantastic@mail.ru
} 
The sample was placed in a thermocell located inside magnetic coils which were fed from the signal generator Agilent 33210a. The amplitude of the pumping field $h$ was varied between $0 \div 20$ Oe and its frequency was $120 \mathrm{~Hz}$. A permanent magnetic field $H$ up to 100 Oe of the same direction was produced by the Helmholtz coils. The sample was blown with a stream of the nitrogen gas, whose temperature was adjusted in the range from $150 \mathrm{~K}$ to $400 \mathrm{~K}$ with an accuracy of 0.1 degree.

The ME signal was taken across the electrodes of the PE layer and applied to the input of the spectrum analyzer SR770. This allowed for simultaneous measuring of all harmonics of the ME voltage.

Figure 2 shows a typical voltage spectrum generated by the LGT-Metglas structure due to nonlinear ME effect at bias field $H=4$ Oe and pumping field $h=5$ Oe. Spectral components with amplitudes $u^{(1)}, u^{(2)}$ and $u^{(3)}$ are clearly visible. Based on the measured spectra, the dependences of the harmonic amplitudes on temperature were plotted for different amplitudes.

\subsection{Measurement results}

Figure 3 shows the temperature dependences of the amplitudes for the first three harmonics of the signal at bias field $H=4$ Oe and two pumping fields $h=5$ Oe and $h=15$ Oe. One should note the different character of the harmonics amplitudes temperature dependences. Thus, for $h=5$ Oe $u^{(1)}$ first grows with temperature, reaches a maximum of $6 \mathrm{mV}$ at $T \approx 220 \mathrm{~K}$, and then decreases again. The amplitude of the 2nd harmonic grows from $u^{(2)}=7 \mathrm{mV}$, reaching a maximum at $T \approx 270 \mathrm{~K}$, while $u^{(3)}$ gradually increases from $2.5 \mathrm{mV}$ at $T=150 \mathrm{~K}$ to $5 \mathrm{mV}$ at $T=300 \mathrm{~K}$.
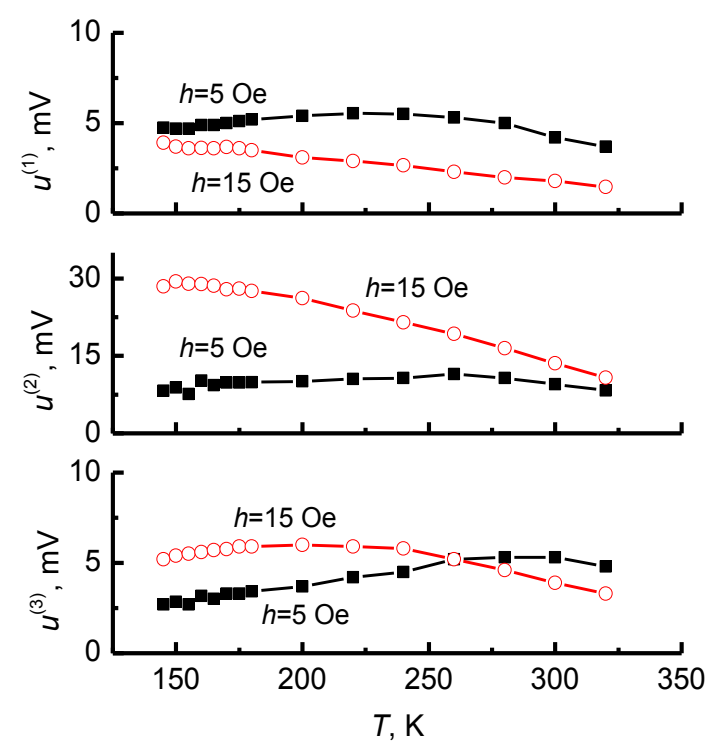

Fig. 3. Amplitudes of three harmonics of the ME signal in the LGT-Metglas structure versus temeprature at $H=4 \mathrm{Oe}$ and pumping fields $h=5$ Oe and $h=15$ Oe.

An increase in the pumping field up to 15 Oe has led to a change in the temperature dependences of the harmonics. The harmonics $u^{(1)}$ and $u^{(2)}$ decrease monotonically by a factor of about 2 at heating from $150 \mathrm{~K}$ to $350 \mathrm{~K}$, and $u^{(3)}$ first increases with temperature, reaches a maximum at $T \approx 200 \mathrm{~K}$, and then falls again.

\section{Discussion}

The form of the temperature dependence of the given ME harmonic can be expressed by the relation [12]:

$$
u^{(n)}(T) \sim \frac{d_{11}(T) q^{(n)}}{\varepsilon(T)},
$$

where $d_{11}$ is the piezoelectric coefficient of the PE layer, $\varepsilon$ is the releative permittivity of the PE layer, $q^{(n)}$ is the piezomagnetic coeffictient for the $n$-th harmonic, whose magnitude is determined by the field dependence of the magnetostriction, the ac field amplitude $h$, and the dc field $H$. It follows that the differences in the character of the temperature dependences of the harmonics are caused by the behavior of the corresponding piezomagnetic coefficient. It has been experimentally established [13], that in the investigated temperature range the ratio $d_{11} / \varepsilon$ for LGT is almost constant.

For the case of small $h$ for a given biasing $H=H_{0}$ the piezomagnetic coefficient is simply the $n$-th derivative of the magnetostriction with respect to the field [8]:

$$
q^{(n)}=\left.\frac{d^{n} \lambda}{d H^{n}}\right|_{H=H_{0}}
$$

where $\lambda$ is the magnetostriction of the FM layer.

In the case of large pumping, expression (2) is inapplicable and piezomagnetic coefficients should be considered as a function of several variables $q^{(n)}(h, H, T)$. Nevertheless, it is obvious that the quantity $q^{(n)}$ in any case is determined by the form of the dependence $\lambda(H)$.

With the help of the LakeShore 7400 vibrating sample magnetometer (Department of Magnetism, Moscow State University), the magnetization curves for the Metglas plate at temperatures from $140 \mathrm{~K}$ to $350 \mathrm{~K}$ have been measured (see Fig. 4).

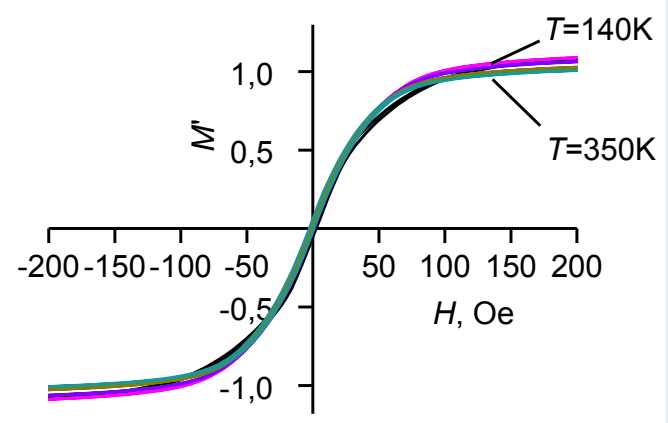

Fig. 4. The magnetization curves of the Metglas plate at different temperatures $T$.

One can see that saturation magnetization decreases by no more than $5 \%$ and some change in the shape of the curve $M(H)$ take place as the temperature increases up to $350 \mathrm{~K}$. In order to characterize more accurately the observed change of the magnetization curve, the graphs of the first three derivatives of magnetostriction $\lambda$ over the 
field $H$ for different $T$ were plotted, assuming that $\lambda \sim M^{2}$ [14] (Fig. 5).

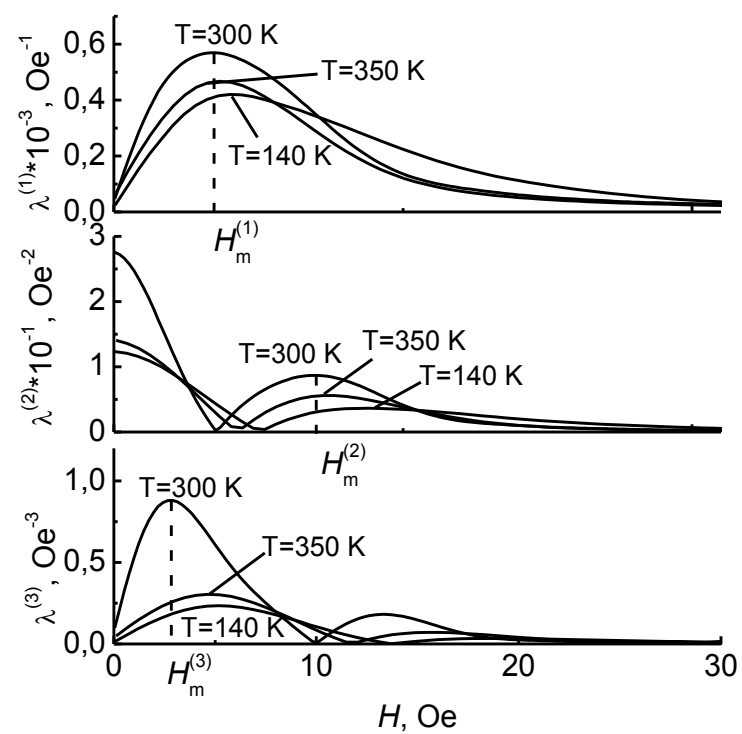

Fig. 5. Field dependences of the derivatives of $M^{2}$ over $H$ at different temperatures.

It is seen that the 1-st derivative increases from zero with increasing $H$, reaches a maximum value $\lambda_{m}^{(1)}$ at $H_{m}^{(1)}$ $\approx 5 \mathrm{Oe}$, and then monotonically drops to zero. The 2 nd derivative has the largest value $\lambda_{m}^{(2)}$ in the zero field, falls with increase of $H$, reaches zero and then has a second maximum at the field $H_{m}^{(2)}$. The 3-d derivative grows from zero with increase of $H$, reaches a maximum value $\lambda_{m}^{(3)}$ for the field $H_{m}^{(3)}$, then drops to zero and has a second maximum.

It can be noted, that all three derivatives change both their maximum values $\lambda_{\mathrm{m}}{ }^{(\mathrm{n})}$ and optimal fields under the influence of temperature

Let's denote the ratio of the maximum value of the $n$ th derivative at a given temperature $T$ to the corresponding value at $T=300 \mathrm{~K}$ as:

$$
\beta^{(n)}=\frac{\lambda_{m}^{(n)}(T)}{\lambda_{m}^{(n)}(T=300 K)} .
$$

And we will characterize the change in the optimal filed $H_{m}^{(n)}(T)$ by the ratio:

$$
\gamma^{(n)}=\frac{H_{m}^{(n)}(T=300 K)}{H_{m}^{(n)}(T)} .
$$

Figure 6 shows the dependences $\beta^{(1)}(T), \beta^{(2)}(T)$, and $\beta^{(3)}(T)$, drawn using the data of Fig. 5 It is seen, that the coefficients $\beta^{(1)}(T), \beta^{(2)}(T)$, and $\beta^{(3)}(T)$ for the Metglas layer posses similar temperature dependences and have a characteristic maximum in visinity of $T \approx 250 \mathrm{~K}$. Wherein, $\beta^{(1)}(T)$ varies by $25 \%, \beta^{(2)}(T)$ - by $50 \%$, and $\beta^{(3)}(T)$ - by $75 \%$ in the investigated temperature range

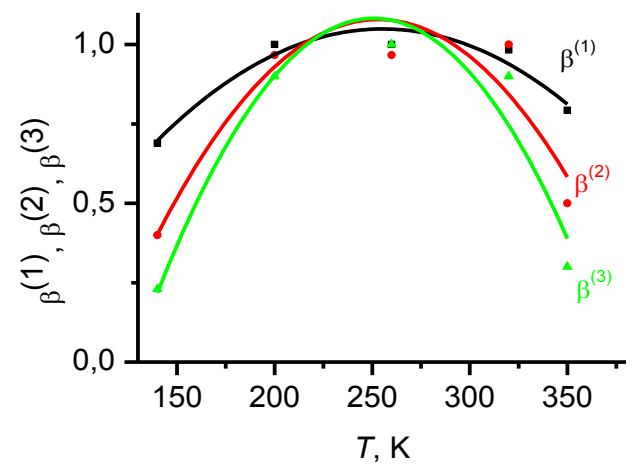

Fig. 6. Temperature dependences of the normalized derivatives of magnetostriction for the Metglas layer. Solid curves are approximations by a $2^{\text {nd }}$ order polynoms.

Figure 7 shows the graphs $\gamma^{(1)}(T), \gamma^{(2)}(T)$, and $\gamma^{(3)}(T)$. It can be seen that optimum fields for all derivatives change almost identically when the temperature is increased from $140 \mathrm{~K}$ to $350 \mathrm{~K}: \gamma$ first increases monotonically from 0.60.7 to 1 , reaching a maximum at $T=270-300 \mathrm{~K}$, and then decreases again to a value of 0.7 .

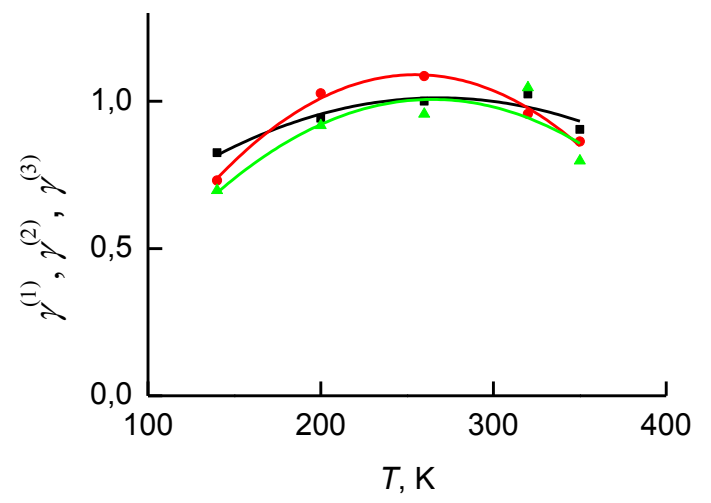

Fig. 7. Temperature dependences of the relative change in the optimal fields for the Metglas layer. . Solid curves are approximations by a $2^{\text {nd }}$ order polynoms.

Let's suggest, that magnetization loop deformation can be expressed as x-axis stretching, which is equivalent to an effective magnetic field change: $H_{\text {eff }}=H \cdot \gamma(T)$. Indeed, if we replace $H$ with $H_{\text {eff }}$ in expression for the piezomagnetic coefficients (2), we obtain:

$$
\begin{aligned}
& q^{(1)}(T, H)=\left.\gamma(T) \cdot \frac{d \lambda}{d H}\right|_{\left(H_{e f f}, T=300 K\right)} \\
& q^{(2)}(T, H)=\left.\gamma^{2}(T) \cdot \frac{d^{2} \lambda}{d H^{2}}\right|_{\left(H_{e f f}, T=300 K\right)} \\
& q^{(3)}(T, H)=\left.\gamma^{3}(T) \cdot \frac{d^{3} \lambda}{d H^{3}}\right|_{\left(H_{e f f}, T=300 K\right)}
\end{aligned}
$$

So, the higher the number of the harmonic, the more sensitive it is to the temperature "compression" of the magnetostriction curve, which is well illustrated by Fig. 5 . Data on Fig.5 and Fig.6 are in a good agreement, which tells that deformation of the magnetization loop and, consequently, deformation of the field dependence of magnetostriction with temperature, can be represented as a compression-stretching along the field axis $H$.

In case of a large pumping amplitude, when the amplitude of the higher harmonics of the ME voltage 
becomes comparable with the amplitude of the first harmonic, then the relations (5-7) for $q^{(\mathrm{n})}(T)$ are no longer satisfied. But they can illustrate how temperature can affect amplitudes of ME signal harmonics in different way.

In other words, effective field change can be presented as equivalent movement left-right on $u(H)$ and $u(h)$ curves, which should be taken in an experiment. As $\gamma(T)$ goes down, our effective field decrease and we move left on $u(H), u(h)$ curves, $\gamma(T)$ increase tends to right-hand movement. As a result nonmonotonic shape of $\gamma(T)$ on Fig.7 dependence can explain the same shape of $u(T)$ curves on Fig.3.

\section{Conclusion}

Thus, it has been shown experimentally that temperature significantly affects the nonlinear ME effect in the two-layer structure of the LGT-Metglas composition, and the character of the temperature characteristics for different ME voltage harmonics differs and depends on the magnitude of the excitation field. The last circumstance introduces a new factor, which should be taken into account when analyzing temperature properties of the ME effect. As far as authors know in all previous studies devoted to this problem influence of ac magnetic field amplitude was totally ignored [4-7]. We suppose that by selecting the appropriate amplitude of the alternating field $h$, it is possible to attain a decrease in the temperature coefficient for a given ME signal, which can be used in the development of new ME devices.

The work was supported by the Russian Science Foundation (agreement 17-12-01435).

\section{References:}

1. C.-W. Nan, M.I. Bichurin, S. Dong, D. Viehland, G. Srinivasan, J. Appl. Phys., 103, p. 031101 (2008).

2. H. Palneedi, V. Annapureddy, S. Priya, J. Ryu, Actuators, 5, No 9, p. 1-31 (2016).

3. J.X. Ye, J.N. Ma, J.M. Hu, Z. Li, M. Feng, Q.M. Zhang and C.-W. Nan C, J. App. Phys. 116, 074103 (2014).

4. F. Fang, Y.T. Xu, W. Yang, J. Appl. Phys. 111, 023906 (2012).

5. Y. Shen, J. Gao, Y. Wang, J. Li, and D. Viehland, Appl. Phys. Lett. 100, 173505 (2012).

6. D. A. Burdin, D.V. Chashin, N.A. Ekonomov, Y.K. Fetisov, Solid State Phenomena pp.233-234.357359 (2015).

7. V.M. Laletin, D.A. Filippov, T.O. Firsova, Tech. Phys. Lett. 40, 3, pp 237-240.

8. D.A. Burdin, D.V. Chashin, N.A. Ekonomov, L.Y. Fetisov, Y.K. Fetisov, G. Srinivasan, G. Sreeniasulu, JMMM, 358- 359, p. 98-104 (2014).

9. Y.K. Fetisov, D.D. Chashin, N.A. Ekonomov, D.A. Burdin, L.Y. Fetisov, IEEE Trans. Magn., 51 (11) 13 (2015).
10. D.A. Burdin, D.V. Chashin, N.A. Ekonomov., Y.K. Fetisov, A.A. Stashkevich, JMMM, 405, p. 244-248 (2016).

11. G. Sreenivasulu, L.Y. Fetisov, Y.K. Fetisov and G. Srinivasan, Appl. Phys.Lett., 100, 05290 (2012).

12. M. Vopsaroiu, J. Blackburn, M.G. Cain, J. Phys. D: Appl. Phys., 40, p. $5027-5033$ (2007).

13. P. Davulis, B.T. Sturtevant, S.L. Duy, M. Pereira, Proc. IEEE Ultrasonics Symposium, p. 1397-1400 (2007).

14. Y.K. Fetisov, D.V. Chashin, N.A. Ekonomov, D.A. Burdin, L.Y. Fetisov, IEEE Trans. on Magn., 51, 11 (2015). 